\title{
Comparative study of a responsive insertion technology (RIT) colonoscope versus a variable-stiffness colonoscope
}

\author{
César Prieto-de-Frías, Miguel Muñoz-Navas, Cristina Carretero, Juan Carrascosa, M. ${ }^{a}$ Teresa Betés,

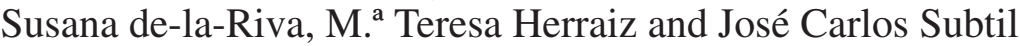

Service of Digestive Diseases. Clínica Universidad de Navarra. Pamplona, Navarra. Spain

\begin{abstract}
Background and objectives: recently, Olympus developed a new prototype (XCF-Q180AY2L) with responsive insertion technology (RIT), which besides the still known variable stiffness technology, included a passive bending section and a new high force insertion tube. Our objective was to investigate whether the use of this prototype could ease colonoscope insertion, increasing the cecal intubation rate, and/or shortening the cecal intubation time.

Material and methods: the study was designed as a prospective observational study in 305 consecutive patients from a colo-rectal cancer screening program. We compared colonoscopies performed with conventional colonoscopes (CFH180AL/CFQ160L) with those performed with the prototype XCF-Q180AY2L. End points were mean cecal and terminal ileal intubation times, cecum intubation rate, and need for specific maneuvers. All colonoscopies were performed under sedation with intravenous propofol. Finally, 288 patients were included.

Results: no complications were observed. Complete cecal intubation rate was $100 \%$ in both groups. The ileum could be reached in $98.95 \%$ of cases. Mean time required to reach the cecum was shorter in the prototype endoscope group (4.31 min, SD $2.63 \mathrm{~min}$ ) than in the conventional endoscope group (4.66 min, SD $2.52 \mathrm{~min}$ ) $(p<0.05)$. Compared with the standard colonoscope group, we observed in the prototype group less subjective sensation of difficulty in the passage of the sigma $(p<0.01)$, fewer maneuvers when it proved necessary to straighten the scope $(p<0.01)$, and less frequent need to modify the stiffness of the endoscope $(p<0.05)$.

Conclusion: we concluded that the prototype endoscope (XCFQ180AY2L) facilitated colonoscope insertion, requiring slightly less time to reach the cecum than a standard colonoscope.
\end{abstract}

Key words: Colonoscope. Prototype. Cecal intubation rate. Cecal intubation time.

\section{INTRODUCTION}

Colonoscopy is currently the method of choice for colorectal cancer screening, for patients older than 50 years, and for people of any age who are at high risk (1). However, for screening programs to be effective, it is essential that endoscopic procedures meet certain minimum quality criteria. One of the most important is that the complete colonoscopy rate should exceed $90 \%$ in all cases, or $95 \%$ of healthy adult screening cases (1). Complete colonoscopy, reaching the cecum, not only depends on the skill of the endoscopist and the sedation depth(2), but also on the technical features of the endoscope (3). With respect to the latter, over the last twenty years, the technical features of endoscopes have greatly improved. Higher image resolution, a wider angle of vision, narrower endoscope shafts, and variable shaft stiffness are some of the many improvements(3).

In 2005, Olympus developed a prototype endoscope (XCF-Q160AW) with a passive bending section (PB), located between the insertion tube and the conventional bending section of the endoscope, to facilitate intubation. A randomized study showed that its use decreased the discomfort of the procedure in on-demand sedation patients (4). These results were reinforced by another study with further advanced colonoscopes in which graded flaccidity of the passive bending section (PB) was introduced (5).

Recently, the same company has developed a new prototype (XCF-Q180AY2L), which, besides the passive bending section (PB), incorporates a new insertion tube that better transmits the pushing force. There are no published data about the impact of using this new insertion tube in real practice, and therefore, neither published studies about the

Prieto-de-Frías C, Muñoz-Navas M, Carretero C, Carrascosa J, Betés MT, De-la-Riva S, Herraiz MT, Subtil JC. Comparative study of a responsive insertion technology (RIT) colonoscope versus a variable-stiffness colonoscope. Rev Esp Enferm Dig 2013;105:208-214.
Correspondence: César Prieto de Frías. Service of Digestive Diseases. Clínica Universidad de Navarra. Avda. Pío XII, 36. 31008 Pamplona, Navarra. Spain e-mail: cprieto@unav.es
Received: 01-10-2012

Accepted: 28-02-2013 
use and possible interaction of these new technologies together (6).

The objective of the study was to compare the time required to reach the cecum, the time required to perform the ileoscopy, and the rate of complete colonoscopies between this new prototype and a conventional colonoscope.

\section{PATIENTS AND METHODS}

\section{The endoscope}

The Olympus prototype XCF-Q180AY2L is similar to the commercially available CFH180AL colonoscope, but differs in terms of two special features.

First, the section immediately proximal to the conventional bending section of the colonoscope is flexible, passively bending itself with the pushing force against a resistance (PB). As this new hyper-flaccid segment bends, the radius of the curve becomes greater, forming an obtuse angle that facilitates the passage of the scope in the flexures, avoiding sharp scope angles that impact the colon wall (Fig. 1). As mentioned above, a previous randomized study showed that its use may facilitate negotiation of sharp bends and reduce patient discomfort (5).

The second special feature is an insertion tube that better transmits the pushing force and torque by reducing the loss of the force at the loop, helping the device to pass the sigmoid colon with less pushing force and less torque (HFT, high force transmission insertion tube) (6) (Fig. 2).

These two incorporations, PB and HFT, in addition to conventional variable stiffness technology, are termed RIT (responsive insertion technology) (Fig. 3). They do not change the external appearance of the endoscope (Table I).

RIT technology similar to that incorporated in this prototype has been included in the new series of Olympus CFHQ190 recently commercialized.

\section{Study design}

Between September and December 2009, 305 consecutive endoscopy unit outpatients who were to undergo colonoscopy

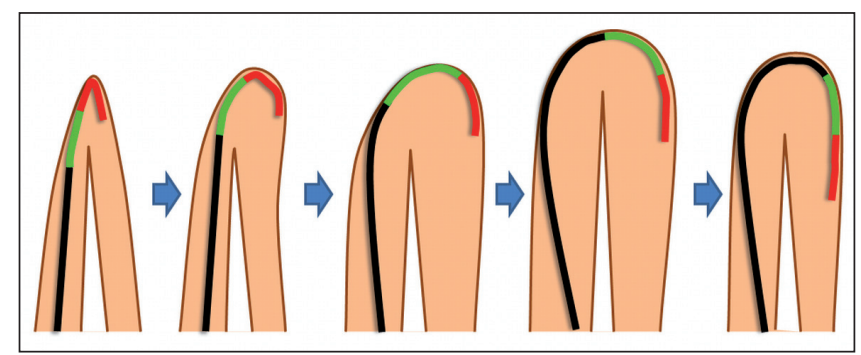

Fig. 1. Passive bending section (PB), with progressive flaccidity facilitates the passage of the tube in the flexures, avoiding sharp tube angles that impact the wall of the colon.

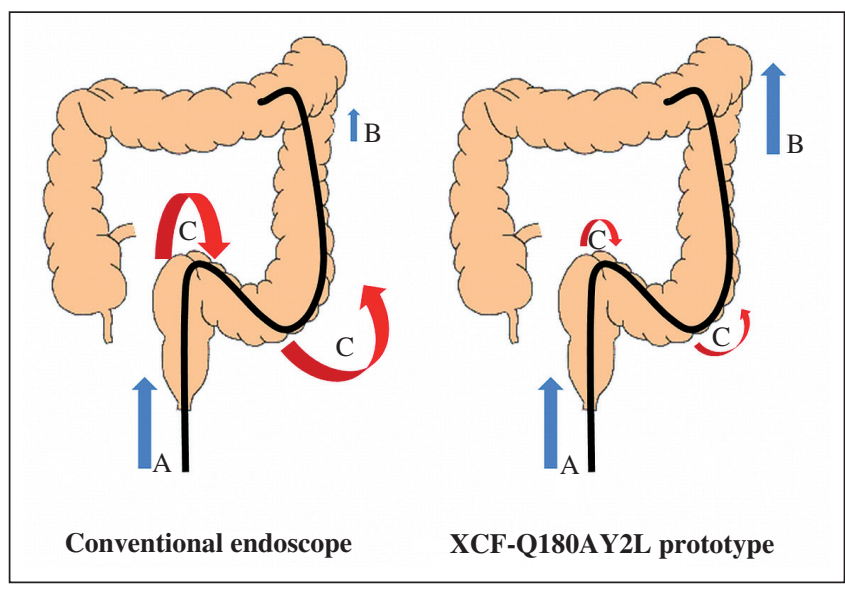

Fig. 2. High force transmission insertion tube (HFT) that better transmits the pushing force and torque by reducing their loss at the loop.

screening for colorectal cancer, were invited to participate in this study upon arrival for their appointment.

Inclusion criteria were that patients were between 18 and 80 years of age, able to give informed consent, whose indication for colonoscopy was colorectal cancer screening, and for whom this was a first or follow-up colonoscopy. We excluded all patients with suspected significant gastrointestinal bleeding, major abdominopelvic surgical history, previous colonic resections, known inflammatory bowel disease or specific conditions that made it theoretically more desirable to use a specific colonoscope (e.g., stenosis, major bleeding), patients with high anesthetic risk (ASA4), pregnant women, patients unable to give informed consent, or those who refused to receive sedation. Patients with history of previous pelvic radiation were not excluded. The study was approved by the local institutional review boards. Written informed consent was obtained from all participants.

After reviewing the exclusion criteria, verifying that the colonoscopy did not need to be performed with a particular endoscope, a prototype XCF-Q180AY2L, or either a CFH180 or a CFQ160 model was selected, exclusively depending on their availability after the endoscope disinfection process.

All endoscopies, which were performed by six experienced staff endoscopists (each with more than 1,000 previous colonoscopies in our unit), were timed. Endoscopy

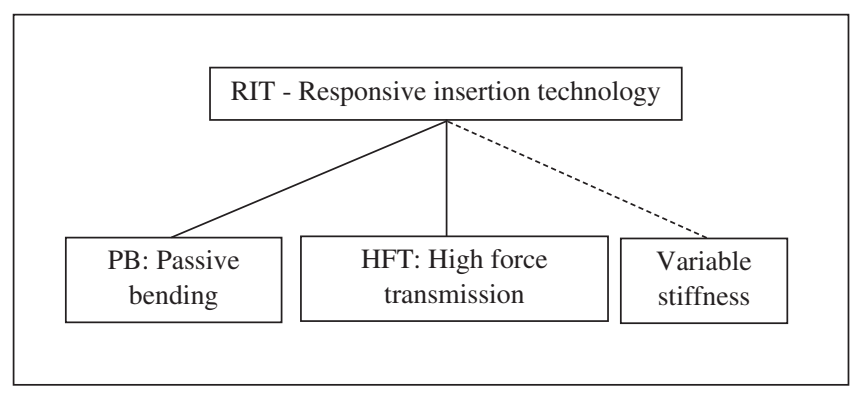

Fig. 3. 
Table I. Characteristics of the prototype and control colonoscopes

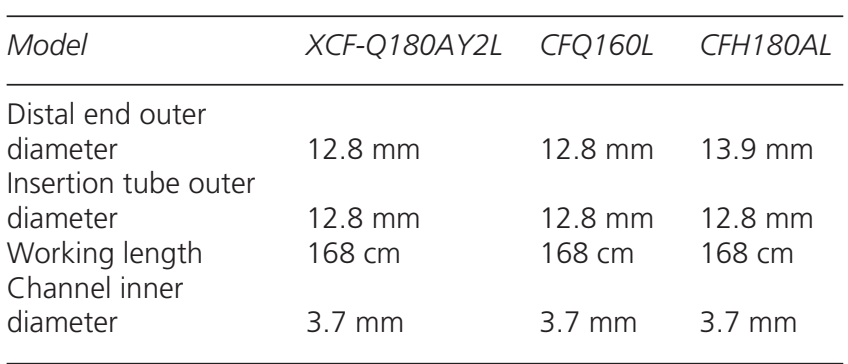

trainees were excluded. All the endoscopists were assisted by nurses, who were responsible for advancing the endoscope during the procedure, as we use to do under routine clinical practice. Each endoscopist worked with the same nurse, in order to minimize interpersonal variability. All colonoscopies were done with the intention of reaching the cecum. Cecal intubation was defined as "passage of the colonoscope tip to a point proximal to the ileocecal valve so that the entire cecal caput, including the medial wall of the cecum between the ileocecal valve and appendiceal orifice, was visible" (1). Once the cecum was reached, ileal intubation was attempted as is done routinely in our unit. If ileal intubation was not achieved after 15 minutes, it was abandoned. All colonoscopies were performed under sedation with intravenous propofol, under the supervision of an anesthesiologist. Obviously, the study could not be double-blinded, as the endoscopist and nurse were both clearly able to distinguish which endoscope they were using. However, the anesthesiologist who administered the intravenous propofol was unaware of the type of endoscope used.

The medication used for colon pre-cleaning and the patient's anesthesia were exactly the same in the two groups, and similar to those of our conventional colonoscopy procedures. Quality of bowel cleansing was graded at the end of the procedure according to the Boston bowel preparation scale (7). The endoscopist was blinded to the type of preparation. There were also no changes in the routine procedures characteristic of our endoscopy unit.

Main objective was to compare mean cecal intubation time, ileal intubation time, and cecum intubation rate, in colonoscopies performed with conventional colonoscopes (CFH180AL/CFQ160L) with those performed with the prototype XCF-Q180AY2L.

Other variables analyzed in the study were: a) the need to use the variable endoscope stiffness function; $b$ ) the need to change the patient position; c) maneuvers needed to straighten the endoscope; and d) the requirement to apply manual pressure to different areas of the abdomen to facilitate endoscope insertion. All these data were collected as dichotomous variables (yes/no), and, where appropriate, the number of times was recorded. We also compared the endoscopist subjective sensation of difficulty on passing or reaching the various sections and flexures of the colon: The sigmoid, splenic flexure, hepatic flexure, cecum, and ileum. These data were recorded as dichotomous variables (easy/difficult). As nurses were the responsible of pushing the endoscope, finally, we also collected as a dichotomous variable (easy/difficult) the nurse subjective sensation of difficulty on performing the complete colonoscopy and the ileoscopy.

\section{Statistical methods}

A 1 minute difference in the cecal intubation time was defined as the threshold for clinical significance. Based on information from fifteen previous patients, we estimated that 63 patients would have to be included in each study arm to detect a 1 minute difference between the two groups in the cecal intubation time at a significance level of $\alpha=0.05$ and a statistical power of $80 \%$. To collect and process data, we used SPSS 15.0. Means of continuous variables that satisfied normality criteria were compared using Student's unpaired samples t-test. Variables that did not meet criteria of normality were compared using the non-parametric Mann-Whitney U test. Categorical variables were compared using the Chisquare test. Statistical significance was concluded for values of $\mathrm{p}<0.05$.

\section{RESULTS}

Finally, 288 patients (179 men, 109 women; mean age 56.9 years) were included in the study. The remaining 17 patients were not included in the study because they did not satisfy the inclusion criteria: 2 because of high anesthetic risk, and 15 because of previous colonic resection. In 138 patients, colonoscopy was performed using the prototype endoscope; while a conventional endoscope was used in the other 150 patients. Of this latter group, the CFH180AL endoscope was used in 120 cases, and the CFQ160AL endoscope was used in 30 cases.

No complications were observed in any of the procedures included in the study. We found no differences in the distribution of sex, age, height, weight, BMI, prevalence of diverticulosis, preparation, cleanliness or previous history of abdominal surgery or pelvic radiation between the groups of patients assigned to the prototype endoscope or conventional endoscopes (Table II). Within the latter group, no differences were found between the patients assigned to the Q160AL or H180AL scopes. All endoscopist and nurse teams used the two endoscopes equally often.

The complete cecal intubation rate was $100 \%$ in both groups. The ileum could be reached in $98.95 \%$ of cases. Ileal intubation was not achieved in 3 of 288 cases ( 2 prototype cases, and 1 control case) in less than 15 minutes, so the maneuver was abandoned, as it was not specifically indicated.

The time required to reach the cecum was significantly shorter in the prototype endoscope group (mean $4.31 \mathrm{~min}$, 
SD $2.63 \mathrm{~min}$ ) than in the conventional endoscope group (mean 4.66 min, SD $2.52 \mathrm{~min})(\mathrm{p}<0.05)$ (Table III). Differences were also statistically significant when comparing the subjective sensation of difficulty in the passage of the sigma. We observed less difficulty with the prototype (13.63 $\%$ difficult cases) than with the conventional endoscope (28.68\% difficult cases). When we compared the need (yes/no) to pull back to straighten the scope after passing a curve, no significant difference was found between the two scopes. However, when this proved necessary, the maneuver was performed significantly less frequently in the prototype group $(\mathrm{p}<0.01)$ (Table III). It was also less frequently necessary to modify the stiffness of the prototype endoscope $(1.4 \%)$ than of the control endoscope $(6.7 \%)$ $(\mathrm{p}<0.05)$ (Table III).

The endoscopist perception of difficulty passing the splenic and hepatic flexure, or of reaching the cecum, was lower with the prototype endoscope than with the conventional one, but these differences were not statistically significant. The time required to perform the ileoscope was shorter and the perceived difficulty of doing it was lower in the conventional endoscope group than in the prototype one, but, once again, the differences were not statistically significant (Table III).

Nurse perception of difficulty performing the complete colonoscopy was also lower with the prototype endoscope than with the conventional one. The difference was statistically significant. Their perceived difficulty to perform the ileoscope was higher in the prototype endoscope group than in the conventional one, but, as occurred with the endoscopists perception, the differences were not statistically significant (Table III).

No significant differences were noted in the other characteristics assessed: The need for manual pressure and changes in patient posture.

In the control group, in which we used a conventional endoscope, we found no statistically significant differences between the CFQ160AL and the CFH180AL series for any of the variables tested.
Table II. Patient characteristics of the prototype and control groups

\begin{tabular}{lll}
\hline & $\begin{array}{l}\text { Prototype } \\
(n=138)\end{array}$ & $\begin{array}{l}\text { Control } \\
(n=150)\end{array}$ \\
\hline Sex (\% males) & $62.3 \%$ & $62.0 \%$ \\
Age (years) & $55.88(12.24)$ & $57.95(13.09)$ \\
Height (m) & $1.68(0.08)$ & $1.68(0.08)$ \\
Weight $(\mathrm{kg})$ & $76.53(14.33)$ & $75.91(12.65)$ \\
BMI (kg/m²) & $26.59(4.66)$ & $26.74(3.57)$ \\
Bowel prep (\% PEG)* & $62.2 \%$ & $63.2 \%$ \\
Bowel cleansing (Boston scale) & $2.30(0.85)$ & $2.38(0.83)$ \\
Diverticulosis (\%) & $21.7 \%$ & $22.6 \%$ \\
Radiotherapy (\%) & $1.4 \%$ & $2.6 \%$ \\
Abdominal Surgery (no colon) $\%$ \%) & $29.7 \%$ & $25.3 \%$ \\
Gynecological surgery (\%) & $8.6 \%$ & $4.6 \%$ \\
\hline
\end{tabular}

Results are means (SDs), or percentages, where specified. There were no statistically significant differences in any comparison. *Only two bowel preparations were used: PEG and NaP. SD: Standard deviation; BMI: Body mass index (kg/m2); PEG: Polyethylene glycol; NaP: Sodium phosphate.

\section{DISCUSSION}

First, we must emphasize the safety of the prototype endoscope, since there were no complications associated with its use in the study.

The complete colonoscopy rate with the prototype endoscope was the same as the control group rate: $100 \%$ in both cases. This result is consistent with some skilled technical colonoscopist reports $(1,8,9)$. Mean insertion time to the cecum with both endoscopes (prototype: $4.31 \mathrm{~min}, \mathrm{SD} 2.63$ min; control: $4.66 \mathrm{~min}, \mathrm{SD} 2.52$ ) was slightly shorter than that reported in most other published studies (10-12), but consistent with (8), or even longer than some other experts studies $(13,14)$. We believe these results may be influenced by the high level of experience of the endoscopists selected for the study $(2,11,15)$, the systematic sedation of all patients $(2,15)$, the exclusion of those patients with previous major abdominal surgery (15), and the "four-handed" way

Table III. Prototype and control group comparison

\begin{tabular}{llll}
\hline & Prototype $(n=138)$ & Control $(n=150)$ & $p$ value \\
\hline Cecal intubation rate (\%) & & & $p>0.05$ \\
lleal intubation rate (\%) & 100 & 100 & $p>0.05$ \\
Cecal intubation time (min) & 98.55 & 99.33 & $p<0.05$ \\
lleal intubation time (min) & $4.31(2.63)$ & $4.66(2.52)$ & $p>0.05$ \\
Rectification (\%) & $1.41(1.64)$ & $1.19(1.30)$ & 74.7 \\
Number of rectifications & 73.7 & $3.28(1.85)$ & $p<0.05$ \\
Stiffness modifications (\%) & $2.56(1.96)$ & 6.7 & $p<0.05$ \\
Endoscopist Sigmoid difficulties (\%) & 1.4 & 28.7 & $p<0.01$ \\
Endoscopist cecal difficulties (\%) & 13.6 & 46.7 & $p>0.05$ \\
Endoscopist ileal difficulties (\%) & 36.8 & 32.7 & $p>0.05$ \\
Nurse difficult colonoscopies (\%) & 35.3 & 30.32 & $p<0.05$ \\
Nurse difficult ileoscopies (\%) & 16.36 & 29.03 & $p>0.05$ \\
\end{tabular}

Results are means (SDs), or percentages, where specified. 
of performing the procedure, as the assistant nurses were responsible for advancing the endoscope (16).

An important finding of this study was that the time needed to reach the cecum was shorter in the prototype endoscope group than in the conventional endoscope group. These differences were small, and therefore of questionable clinical importance, but they are obtained against two of the most advance conventional endoscopes currently available, and consistent with the trend shown in two previous studies in which endoscopes with passive bending sections were tested $(4,5)$. The subjective feeling of difficulty in passing the sigma, and the need to use the variable stiffness function of the endoscope, were also lower in the prototype group. Both findings are consistent, as it is in the sigmoid colon, with its characteristics acute angles and slack and longer mesenterium, where the advantageous technical features of the prototype would be most useful. The new PB section of the prototype should transform these sharp angles into obtuse ones, decreasing both the impact of the endoscope against the wall and the consequent stretching. Also, the pushing force and torque should be more effectively transmitted to the tip of the endoscope, facilitating the passage through the sigmoid colon with lower pushing force and torque. These advantages of the prototype may also be effective in other sections of the colon, but as these sections present intrinsically a lower technical demand, the advantages of the prototype are not likely to be so apparent. We believe that is the main reason why the comparative difficulty in passing other sections of the colon, although favorable to the prototype endoscope, was not statistically significant.

No differences were found between the two groups when comparing the need to pull back to straighten the colon during the procedure. However, when it was necessary, significantly fewer maneuvers were made with the prototype endoscope group than in the conventional endoscope group. This difference suggests that when colonoscopy was technically more complicated (with more angles and a tendency to form loops and overstretch) the endoscope prototype was more useful.

An important detail is that more time was needed to perform the intubation of the ileum, and the subjective feeling of difficulty was greater, in the endoscope prototype group than in the control group, although neither difference was statistically significant. Kessler et al. concluded in a previous study (14) that short bending colonoscope sections facilitated proximal colon retroflexion but could hamper terminal ileal intubation in pediatric colonoscopies. Long bending sections would deflect off the lateral wall of the cecum, increasing the transmission of direct force to the tip, thereby advancing more deeply into the ileum. Although this observation appears to contradict our results, we suspect that the prototype's longer flexible end section, as well as the fact that it passively bends with the pushing force against a resistance (in this case, the ileocecal valve), could reduce its maneuverability in the cecum, hampering the ileoscopy.
As nurses were responsible of pushing the endoscope, we thought it would be an important fact to know their subjective perception of difficulty on advancing the new endoscope. Nurses collected data were quite similar to the endoscopist's ones, which, in our opinion, reinforce the conclusions.

A criticism of the study is that, due to the nature of the test, it could not be double-blinded. Both the endoscopist and the endoscopist nurse knew with which colonoscope they were performing the test, which could have biased the results, since they could have improved their technique and been more careful at the angles when using the prototype endoscope. However, we believe that the $100 \%$ complete colonoscopy rate suggests an adequate technique irrespective of the scope used. Moreover, the fact that ileoscopy was more difficult to perform with the prototype endoscope, even given the potential advantage of commitment and care when using it, argues against that suggestion. In any case, we tried to avoid most of the other possible biases: The anesthesiologist was unaware of the endoscope used, so any differences could not have been due to the depth of sedation. Also, the variations in the anthropometric characteristics of patients, the preparation regimen and cleanliness were homogeneous in the two groups, with no significant differences.

Another valid criticism is the lack of formal randomization of the patients. However, we believe that selecting endoscopes entirely on the basis of their availability after the disinfection process, ensured their random assignment to the patients.

Hoff et al. have recently published a study with a similar, but not identical prototype (5). Although both colonoscopes incorporated the PB section, the prototype used in our study adds a high force transmission tube (HFT), which becomes stiffer from a more proximal point when the variable stiffness function is used. In addition, the primary objectives differed radically, as in the cited study the primary endpoint was the patients' evaluation of pain, while in our study all the procedures were done under deep sedation (so patient comfort could not be measured) and the primary endpoints were cecal intubation rate and time. Nevertheless, both studies showed shorter times to reach the cecum when using the prototype, making the results more consistent.

In conclusion, the prototype XCF-Q180AY2L endoscope was as safer as the conventional colonoscopes used in our endoscopy unit (CFH180AL and CFQ160L). Its use allowed to reach a cecal intubation rate as high as the obtained in the control group, which was $100 \%$. It slightly shortened the time taken to reach the cecum. Time differences were small and therefore, of doubtful clinical relevance, nevertheless, they were statistically significant, obtained against two of the most advance conventional endoscopes currently available, and consistent with the trend shown in the two previous studies in which passive bending sections were tested. Prototype XCF-Q180AY2L may facilitate the endoscope procedure in difficult colonoscopies, since it use decreased not only the number of times 
we needed to straight the scope after passing a curve when this proved necessary, but the need to modify the stiffness of the colonoscope. Endoscopists and nurses subjective impression was that the prototype eased the passage of the sigma. It is possible that its technical characteristics may reduce its maneuverability in the cecum, increasing the difficulty of achieving the ileoscopy. However, we believe the results we have obtained with this prototype are promising, and that the introduction of these new technical improvements in future colonoscope models may eventually ease the procedures. Performing a similar study with more patients, more complex colonoscopies, and perhaps using magnetic positioning imaging systems (such as ScopeGuide ${ }^{\circledR}$ ), might confirm our results and determine whether the prototype technical features affect the ileal intubation rate.

\section{REFERENCES}

1. Rex DK, Petrini JL, Baron TH, Chak A, Cohen J, Deal SE, et al. Quality indicators for colonoscopy. Gastrointest Endosc 2006;63(Supl. 4);S1628.

2. Cardin F, Minicuci N, Andreotti A, Pinetti E, Campigotto F, Donà BM, et al. Maximizing the general success of cecal intubation during propofol sedation in a multi-endoscopist academic centre. BMC Gastroenterol 2010;10:123.

3. Cappell MS, Abboud R. The impact of advances in instrumentation and techniques of colonoscopy from 1988 to 2008 on inpatient colonoscopy performance at a high volume endoscopy unit in the United States: Significantly shorter procedure time, higher completion rate, performance on sicker inpatients, and near disappearance of flexible sigmoidoscopy. Dig Dis Sci 2010;55:3521-9.
4. Hoff G, Bretthauer M, Huppertz-Hauss G, Sauar J, Paulsen J, Dahler $\mathrm{S}$, et al. Evaluation of a novel colonoscope designed for easier passage through flexures: A randomized study. Endoscopy 2005;37:1123-6.

5. Hoff G, Moritz V, Bretthauer M, Ludolph T, Huppertz-Hauss G, Paulsen J, et al. Colonoscope with a sub-distal hyper-flaccid segment for improved insertion at colonoscopy: A randomized study. Scand J Gastroenterol 2011;46:104-8.

6. Saito Y, Kimura H. Responsive insertion technology. Dig Endosc 2011:23(Supl. 1):164-7.

7. Lai EJ, Calderwood AH, Doros G, Fix OK, Jacobson BC. The Boston bowel preparation scale: A valid and reliable instrument for colonoscopy-oriented research. Gastrointest Endosc 2009;69(3 Pt 2):620-5

8. Hansen JJ, Ulmer BJ, Rex DK. Technical performance of colonoscopy in patients sedated with nurse-administered propofol. Am J Gastroenterol 2004;99:52-6.

9. Leung FW. Methods of reducing discomfort during colonoscopy. Dig Dis Sci 2008;53:1462-7.

10. Nelson DB, McQuaid KR, Bond JH, Lieberman DA, Weiss DG, Johnston TK. Procedural success and complications of large-scale screening colonoscopy. Gastrointest Endosc 2002;55:307-14.

11. Bernstein C, Thorn M, Monsees K, Spell R, O'Connor JB. A prospective study of factors that determine cecal intubation time at colonoscopy. Gastrointest Endosc 2005;61:72-5.

12. Kim WH, Cho YJ, Park JY, Min PK, Kang JK, Park IS. Factors affecting insertion time and patient discomfort during colonoscopy. Gastrointest Endosc 2000;52:600-5.

13. Deenadayalu VP, Chadalawada V, Rex DK. 170 degrees wide-angle colonoscope: Effect on efficiency and miss rates. Am J Gastroenterol 2004;99:2138-42.

14. Kessler WR, Rex DK. Impact of bending section length on insertion and retroflexion properties of pediatric and adult colonoscopes. Am J Gastroenterol 2005;100:1290-5.

15. Harris JK, Froehlich F, Wietlisbach V, Burnand B, Gonvers JJ, Vader JP. Factors associated with the technical performance of colonoscopy: An EPAGE Study. Dig Liver Dis 2007;39:678-89.

16. Hoff G, Volker M, Bretthauer M, Aabakken L, Høie O, Delange T, et al. Gastronet survey on the use of one- or two-person technique for colonoscopy insertion. BMC Gastroenterol 2011;11:73. 